\title{
The Cyborg Method: A Method to Identify Fraudulent Responses From Crowdsourced Data
}

*For correspondence:

Matthew.Price@uvm.edu (MP)

Present address: 2 Colchester Ave, Burlington VT 05405

\section{Matthew Price $^{1 *}$, Johanna E. Hidalgo ${ }^{1}$, Julia Kim ${ }^{1}$, Alison C. Legrand ${ }^{1}$, Zoe M. F. Brier $^{1}$, Amy Hughes Lansing ${ }^{2}$, Ateka A. Contractor ${ }^{3}$}

${ }^{1}$ Center for Research on Emotion, Stress, and Technology, Department of Psychological Science, University of Vermont; ${ }^{2}$ Department of Psychological Science, University of Vermont; ${ }^{3}$ Department of Psychology, University of North Texas
Abstract Crowdsourcing has become an essential data collection method for psychological research, especially when access to pools of participant are limited by location and resources. Concerns about the validity and quality of crowdsourced data persist, however. A recent documented increase in the number of invalid responses within crowdsourced data has highlighted the need for quality control measures. Although a number of approaches are recommended, few have been empirically evaluated. The present study evaluated a Cyborg Method that used automated evaluation of participant meta-data and a review of short answer responses. Two samples were recruited - in the first, the Cyborg Method was applied after data collection to gauge the extent to which invalid responses were collected when a priori quality controls were absent. In the second, the Cyborg Method was applied during data collection to determine if the method would proactively screen invalid responses. Results suggested that Cyborg Method identified a substantial portion of invalid responses and both automated and human evaluation components was necessary. Furthermore, the Cyborg Method could be applied proactively to screen invalid responses and substantially reduced the per participant cost of data collection. These results suggest that the Cyborg Method is a promising means by which to collect high quality crowdsourced data.

\section{Implementing the Cyborg Method}

Details how to implement this method are available here https://www.crestresearch.org/ research/cyborg-method

This manuscript is under review.

\section{Introduction}

Crowdsourcing platforms such as Amazon's Mechanical Turk and Prolific are widely used to collect data for psychological research (Buhrmester et al., 2018). The number of published stud- 
ies that used these platforms has increased 10 -fold in the past decade (Mellis Bickel, 2020). There are several reasons for this rapid increase: data collection via self-report surveys and experimental methods, ease of recruitment, access to hard-to-reach populations, and the relatively low cost (Arditte et al., 2016; Shapiro et al., 2013; Strickland Stoops, 2019; Weber, 2021). There are perennial concerns, however, regarding the validity of crowdsourced data (Chandler et al., 2020; Shapiro et al., 2013). These concerns have grown in recent years due to several documented cases in which large portions of responses in crowdsourced datasets were deemed invalid (Chandler et al., 2020; Dennis et al., 2019). Given the potential benefits of crowdsourced data collection, countermeasures are needed for this type of data collection to remain viable.

Concerns about the quality of crowdsourced data have grown with recent reports of data contaminated with invalid responses (Chandler et al., 2020; Dennis et al., 2019). The invalid resposnes were detected when conducting the primary outcome analyses and the investigators obtained markedly different results than what was hypothesized. They carefully inspected the datasets and discovered several responses answered self-report measures seemingly at random. These invalid responses significantly differed from valid responses across multiple measures of psychopathology. Furthermore, well-established associations between psychopathology and other constructs, such as social support, differed between valid and invalid responses as well. Without proper detection, these invalid responses would have led to incorrect results and conclusions being drawn.

The inclusion of invalid responses also placed a substantial financial burden on these research studies. Most studies use a priori power analysis to determine the required sample size to be recruited. This sample size dictates the budget required to complete the study. When a portion of the compensated responses are invalid, however, the target sample size may not be reached. Thus, valid conclusions from the collected data cannot be drawn (Chandler et al., 2020). Given the potential risks of collecting and using invalid responses from crowdsourced data, there is a need for quality control methods to maintain the feasibility of such research projects. Although basic quality control methods to screen for such invalid responses are integrated into many survey platforms (Qualtrics XM, 2021), increasingly sophisticated strategies are used by a subset of participants to circumvent these protections. These strategies include the use of Virtual Private Networks (VPNs) or Virtual Private Servers (VPSs) and bots (Dennis et al., 2019). VPNs allow an internet connection to appear as if it has originated from a different location. Many crowdsourced studies restrict access to those within a particular country (Boas et al., 2020). The user's country is determined using the device's Internet Protocol (IP) address. VPNs alter the IP address so the device appears as if it is in a location allowed by the study, thus giving study access to individuals outside of the target country. Bots are the use of automated software to complete surveys or experiments in a manner that mimics a trauma. Bot behavior may include automatically completing survey responses at a pre-specified rate, selecting responses based on a pre-specified pattern, and inserting short written responses or narratives that are gleaned from internet search engines. These strategies allow studies to be completed with invalid responses that advanced methods to detect. Several recent investigations have recommended quality control methods. One method is IP evaluation (Aguinis et al., 2021; Chandler et al., 2020; Dennis et al., 2019, 2019; MacInnis et al., 2020). IP evaluation uses 3rd party services that compare an IP address against a list of addresses known to be used by VPNs. VPNs will often assign users to a set of addresses affiliated with a given location, making it possible to flag them as suspicious. This evaluation can be done post-hoc if IP addresses were collected or in real time via an application programming interface (API) embedded within the survey platform. A recent comparison between those who were flagged as using a VPN and those who were not demonstrated that these services can accurately identify invalid responses (Dennis et al., 2019). In this study, individuals who used a VPN were more likely to fail a question that requested a specific answer (e.g., "For this question, please answer none of the above"), provided lower quality 
responses to short answer questions, and were more likely to provide nonsensical responses to short answer questions. Despite the promise of IP evaluation to identify fraudulent respondents, it has several limitations (Dennis et al., 2019). First, IP evaluation is performed by 3rd party companies who use proprietary methods that are not subject to independent evaluation by researchers. Second, IP address identification occurs at the location of the device. It may incorrectly flag multiple users in a similar location, such as multiple individuals who complete the study while in the same location, as fraudulent. Furthermore, the manner in which internet service providers (ISPs) assign IP addresses may lead an IP evaluation service to label an IP as problematic incorrectly. For example, an ISP can use a dynamic IP address that changes routinely such that it can be difficult to determine if a given user is using a VPN. Finally, certain ISPs may use network address translation (NAT) that allows multiple users in a similar location to share an IP address. These IP addresses are more likely to be flagged as VPNs because of the number of users associated with a single address. These limitations suggest that additional measures are needed beyond IP address evaluation. A second recommendation for identifying invalid responses is embedding items that require a specific response and then determining the number of responses that met these criteria. The most common methods are "attention-check" or "knowledge-check" items. Attention-check items ask for a specific response (e.g., "Choose option 3") or ask about whether fictional events have occurred (e.g., "Have you conducted business in Wakanda?"). A knowledge-check item is one that asks for information that would only be known by a member of a target population (e.g., specific military information for a veteran-specific sample) (Weiss et al., 2021). This approach has been used to identify potential fraudulent responses as well as those who are inattentive (Natesan Batley et al., 2021; Shapiro et al., 2013; van Stolk-Cooke et al., 2018). This method also has limitations. First, these items add to the length of the survey. Second, items that ask about fictional information may confuse participants (e.g., unsure if Wakanda is a real location). The additional time spent on these items may contribute to fatigue or inattention. Indeed, some have cautioned against using these items because they may compromise the data obtained from valid participants (Vannette, 2017). Third, the respondents who provide invalid data may be aware of these items and correctly answer these questions. In one study, half of the invalid responses answered attention-check questions correctly (Dennis et al., 2019). Thus, the use of these questions in isolation is likely insufficient (Agley et al., 2021). A combination approach that integrates an automated evaluation coupled with a researcher's review of responses is recommended (Chandler et al., 2020). This Cyborg Method, in which automated and human evaluation of the data are combined, allows the strengths of each method to offset the limitations of the other. An automated IP evaluation can rapidly identify a invalid responses, thus reducing researcher burden. Those that pass this review then have a set of responses reviewed by investigators. Prior work has suggested that short answer responses are ideal for this purpose (Dennis et al., 2019). Short answer questions can be brief, problematic responses are easily identified, and are difficult for a bot to falsify. It is recommended that the short answer questions ask for a brief description of a personal event or belief (Dolan et al., 2020), which is simple for a valid participant to complete, but would pose a significant challenge for a bot. For example, in conducting a study on individuals who have experienced traumatic events, respondents could be asked to describe their most salient trauma. This line of questioning is part of several well-established measures such as the Life Events Checklist (Weathers et al., 2013). This combined approach of automated review of IP addresses and review of written responses has the potential to efficiently increase data quality. To-date, only a single study has evaluated the benefits of this combined approach (Agley et al., 2021). A large cohort of respondents were randomized to four conditions: no control methods, a bot/VPN evaluation that asked participants a fact-based question that was unrelated to the study, an attention check question that asked about fictional events, and a combination arm that included both the bot/VPN check and the attention check. All arms completed measures about psychopathology as well. It should be noted that the VPN evaluation method was more consistent with a knowledge-check method as opposed to the IP evaluation described above. The results showed that the arm without any control methods had significantly 
higher scores on all measures of psychopathology. This arm had more invalid responses as well. The combination arm had the greatest proportion of valid responses. There were several limitations, however. First, all quality control methods were based on self-report information including the VPN evaluation. Second, participants were compensated with less than the current market rate. Finally, recruitment occurred under a target sample size rather than according to a set budget, which makes it difficult to determine the extent that using this approach reduced overall study costs. That is, recruitment occurred without regard for the cost per participant. Given these limitations and the overall lack of empirical work evaluating quality control methods for crowdsourced data, more work is warranted. The present study evaluated the utility of a Cyborg Method that involved automated IP evaluation and a human review of short answer responses. The primary aim was to determine if these methods improved the quality of crowdsourced data. This aim was evaluated by comparing responses identified as valid to those as invalid. It was hypothesized that both components of the Cyborg Method would be needed as opposed to just one component. A secondary aim was to determine the extent to which these methods reduced study costs. Finally, the classification of valid and invalid responses between the Cyborg Method and the attentioncheck method was compared based on the popularity of the attention check method. It was hypothesized that the Cyborg approach would correctly identify a greater proportion of responses as invalid than attention-check questions alone.

\section{Methods}

The current study included two samples recruited sequentially to avoid participant overlap. For Sample 1, the Cyborg Method was applied after data collection and all participants were compensated. For Sample 2, the Cyborg Method was enforced during data collection. The budget for Sample 1 was 4,900and forSample2was1,331. The budget for Sample 1 was larger to allow for sufficient valid and invalid respondents to be collected. Data collection continued until the budget for each sample was exhausted. All study procedures were approved by the local Institutional Review Board.

\section{Recruitment}

Recruitment occurred via Amazon's Mechanical Turk. Sample 1 data was collected from January-March 2021. Sample 2 data was collected from April-May 2021. For both waves, Human Intelligence Tasks (HITs) were made available to those with a HIT approval rate $>95 \%$ and United States residency. HITs advertised a research study that examined reactions to stressful events. Inclusion criteria for both samples were having experienced directly or witnessed in person a traumatic event that met Criterion A for a posttraumatic stress disorder (PTSD) and residing in the United States (American Psychiatric Association, 2013). The requirement of a traumatic event that met Criterion A was used to collect a personalized writing sample about a traumatic event. Participants were compensated $\$ 4.00$ for successful completion of the HIT. Amazon charged $\$ 0.80$ per participant as a service fee. Thus, the base cost per participant was $\$ 4.80$.

\section{Measures}

Patient Health Questionnaire-8 (PHQ-8; Kroenke, Strine, Spitzer, Williams, Berry, Mokdad, 2009): The PHQ-8 is validated measure of depression symptoms. The PHQ-8 is an 8-item self-report measure that assesses depression symptoms experienced over the past two weeks on a 0-3 point Likert scale. The PHQ-8 is adapted from the PHQ-9 and removes an item on suicidal ideation. The PHQ-8 has good psychometrics (Kroenke et al., 2009). 
Life Events Checklist for the DSM 5 (LEC-5; Weathers, Blake, Schnurr, Kaloupek, Marx, Keane, 2013): The LEC-5 is a 17-item self-report measure that assesses exposure to potentially traumatic events across one's life span. Participants reported exposure to 16 known events, with an additional item included to assess exposure to other extraordinary stressful events. The extended version of the LEC, which asked participants to describe the worst event was used. The extended version of the LEC- 5 was used in the current study, which asked for a written description of the worst event in part 2.

PTSD Checklist for the DSM-5 (PCL-5; Weathers, Litz, Keane, Palmieri, Marx, \& Schnurr, 2013): The PCL-5 is a 20-item self-report measure that assesses PTSD symptoms experienced over the last month. The PCL-5 was anchored to the traumatic event specified in the LEC-5. Responses are provided on a 0-4 point Likert scale. The PCL-5 has excllent psychometrics (Bovin et al., 2015).

Quality Control Measures: Three quality control measures implemented in both samples: (1) Automated IP Evaluation, (2) Attention Review Items, (3) Human Response Review. Information on how to implement these methods is detailed here: https://www.crestresearch.org/research/ cyborg-method.

Automated IP Evaluation: Automated IP evaluation services were used to determine if the IP address of the participant was associated with the use of a VPN or a BOT network. Two external services were used - IPhub (https://iphub.info/) and IPQualityscore (https://www.ipqualityscore.com/). These services maintain an active blacklist of IP addresses associated with problematic internet behavior. They were selected because they performed well in prior studies in which IP evaluations were used (Dennis et al., 2019), have API's that can be integrated into Qualtrics so that IP evaluation can be done automatically (see supplemental materials), and offer free accounts with a monthly quota.

Attention Items: Six attention items were placed throughout the surveys that required participants to provide a specific response (e.g., For this question, please select "Quite a bit."). They were located at equidistant points throughout the survey. A valid response based on the attention check items required at least 5 correct responses.

Short Answer Review: The written response to the description of the trauma in the LEC-5 Part 2 was reviewed by trained research assistants. Instructions for the description question on the LEC-5 asked participants to provide 2-3 sentences describing the most distressing traumatic event to elicit a sufficient writing sample. Participants were also instructed not to include potentially identifying information about their event (e.g., dates, names, locations). The content provided was reviewed to determine if the response described an actual traumatic event. For example, a valid response would entail a description of a car accident that resulted in an injury. An invalid answer would entail text copied from the first hit of a google search on "serious accident or injury" or text taken from a blog post on the internet.

\section{Procedure}

Sample 1. After selecting the HIT, participants completed a consent form and were informed that their IP address would be checked to determine if they were using a VPN. Their IP information was sent to IPhub and IPqualityscore via an integrated API. Information about the likelihood of bots, VPNs, and other VPN behavior was collected, but not used to exclude prospective participants. Those who endorsed experiencing or witnessing an event in person on the LEC-5 were allowed to proceed. Participants then completed all remaining surveys and validity questions. All participants in Sample 1 were compensated. After data collection was completed, written responses for LEC-5 underwent a Short Answer Review. 
Sample 2. Sample 2 data collection proceeded similar to that of Sample 1, except that the quality control measures were enforced while the data was collected. After providing consent and being notified that their IP address would be reviewed, IP addresses were sent to IPhub and IPqualityscore. Those with an invalid IP were discontinued. Those with valid IP's were allowed to complete all remaining measures. Upon completion, two trained research assistants conducted the Short Answer Review of the LEC-5 part 2 and determined the validity of the short answer response. Agreement among the research assistants was 100For Sample 2, participants who were identified as invalid by either method were notified and given the opportunity to appeal this decision. The appeal process for those who had an invalid IP address involved contacting the research team via e-mail and providing information as to their physical location and confirming that they did not use a VPN or Bot. Individuals who appealed were provided separate version of the survey. These responses were then reviewed and compensated accordingly. Seven individuals appealed successfully and were included in the final dataset for Sample 2.

\section{Results}

For Sample 1, $n=4427$ users accessed the HIT 1). Of these responses, $n=2942$ did not endorse an event on the LEC-5 and thus were discontinued for not meeting the inclusion criteria. Of note, a substantial portion of these responses $(n=1815,61.7 \%)$ had invalid IP's. Of the 1485 remaining, approximately a third discontinued voluntarily ( $n=474,31.9 \%)$. The 1011 responses from participants who endorsed experiencing a traumatic event on the LEC-5 and who completed the study were evaluated via the Cyborg Method. Among this group, $n=324,32.0 \%$ were invalid based on their IP address and their short answer response, $n=431,42.6 \%$ had valid IP addresses, and $n=520,51.4 \%$ provided valid short answer responses. A subset, $n=264,26.1 \%$, had a valid IP address and passed the Short Answer Review (Supplemental Tables 1).

Figure 1. Flow diagram illustrating the number of participants screened at each stage of the screening procedures for Sample 1 and Sample 2.
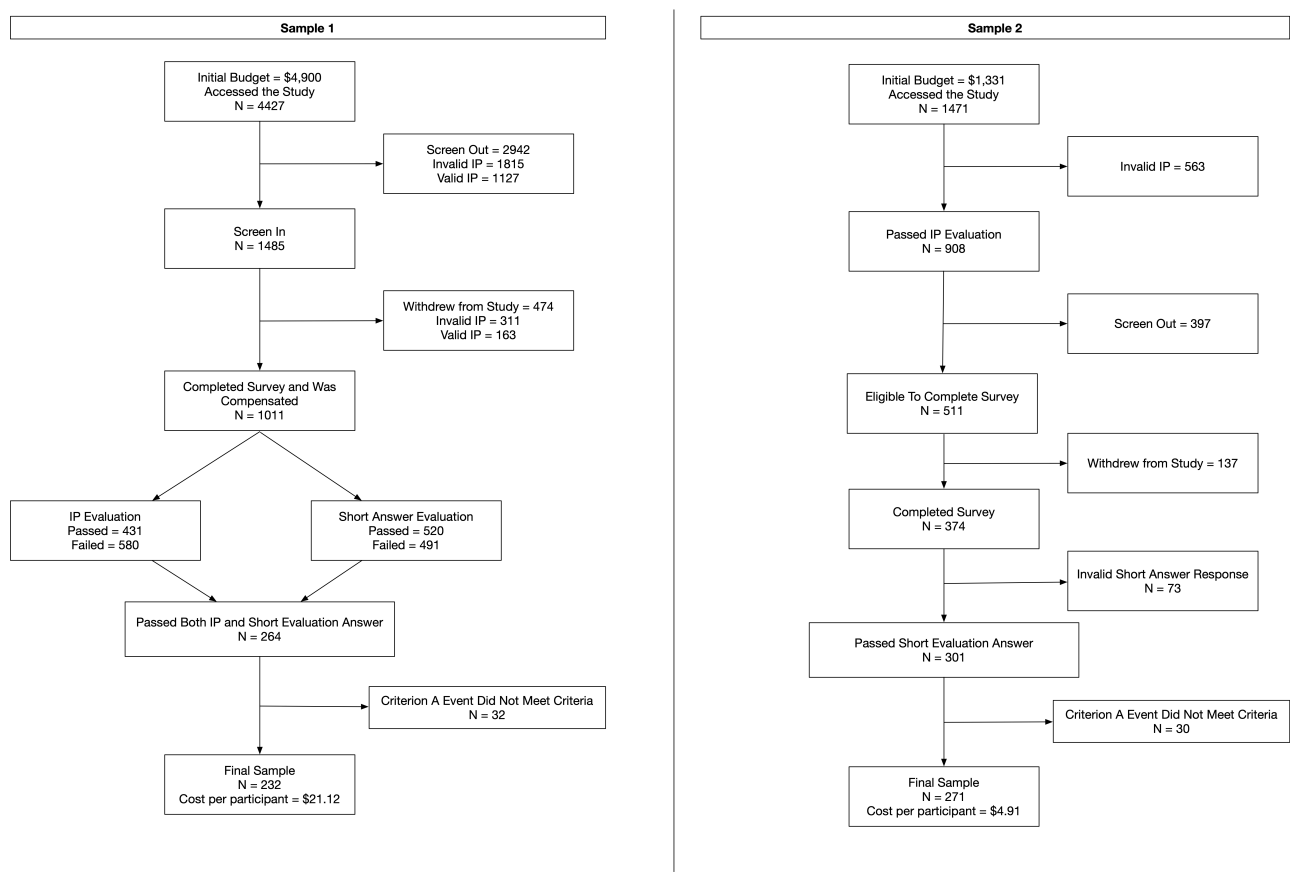

Follow-up analyses were conducted to determine if there were differences in PCL-5, PHQ8, and LEC Trauma types for each component of the Cyborg approach (Table 1). On the PCL-5, there 
Table 1. Descriptive statistics for the valid and invalid responses from Sample 1 and 2.

\begin{tabular}{|c|c|c|c|c|c|c|c|c|}
\hline \multirow[t]{3}{*}{ Variable } & \multicolumn{6}{|c|}{ Sample 1} & \multirow{2}{*}{\multicolumn{2}{|c|}{$\begin{array}{l}\text { Sample } 2 \\
\text { Invalid } \\
N=79\end{array}$}} \\
\hline & \multicolumn{2}{|c|}{$\begin{array}{c}\text { Valid } \\
N=251\end{array}$} & \multicolumn{2}{|c|}{$\begin{array}{l}\text { Invalid } \\
N=732\end{array}$} & \multicolumn{2}{|c|}{$\begin{array}{c}\text { Valid } \\
N=274\end{array}$} & & \\
\hline & M & SD & $\mathrm{M}$ & SD & $\mathrm{M}$ & SD & $\mathrm{M}$ & SD \\
\hline PCL-5 & 27.15 & 19.27 & 43.64 & 16.35 & 21.12 & 19.83 & 41.97 & 23.33 \\
\hline PHQ-9 & 7.6 & 6.08 & 11.97 & 5.03 & 6.56 & 6.59 & 12.49 & 6.85 \\
\hline \multicolumn{9}{|l|}{ LEC- 5 Counts } \\
\hline In Person & 3.08 & 2.60 & 5.85 & 5.34 & 2.61 & 1.82 & 3.65 & 3.72 \\
\hline Witnessed & 1.59 & 2.16 & 2.71 & 3.00 & 1.69 & 2.05 & 3.51 & 2.92 \\
\hline Learned About & 2.12 & 2.89 & 1.82 & 2.52 & 2.85 & 3.22 & 3.67 & 3.33 \\
\hline Exposure Via Work & 0.20 & 0.71 & 0.56 & 1.35 & 0.36 & 0.94 & 1.34 & 1.94 \\
\hline Did Not Experience & 9.01 & 4.89 & 5.04 & 5.88 & 8.49 & 5.08 & 3.83 & 5.43 \\
\hline \multirow[t]{2}{*}{ Age } & 36.09 & 9.89 & 32.40 & 8.07 & 40.07 & 11.71 & 36.51 & 8.08 \\
\hline & $\mathrm{N}$ & $\%$ & $\mathrm{~N}$ & $\%$ & $\mathrm{~N}$ & $\%$ & $\mathrm{~N}$ & $\%$ \\
\hline Gender (Male) & 113 & 45.0 & 400 & 54.6 & 142 & 51.8 & 40 & 50.6 \\
\hline Latinx & 22 & 8.8 & 194 & 26.5 & 37 & 13.5 & 22 & 27.8 \\
\hline \multicolumn{9}{|l|}{ Race } \\
\hline White & 188 & 74.9 & 447 & 61.1 & 207 & 75.5 & 40 & 50.6 \\
\hline African American & 15 & 6.0 & 103 & 14.1 & 28 & 10.2 & 18 & 22.8 \\
\hline American Indian & 4 & 1.6 & 24 & 3.3 & 3 & 1.1 & 1 & 1.3 \\
\hline Asian American & 11 & 4.4 & 139 & 19.0 & 18 & 6.6 & 2 & 2.5 \\
\hline Pacific Islander & 0 & 0 & 8 & 1.1 & 1 & 0.4 & 0 & 0 \\
\hline Bi-racial & 7 & 2.8 & 6 & 0.8 & 7 & 2.6 & 0 & 0 \\
\hline Other & 1 & 0.4 & 5 & 0.7 & 10 & 3.6 & 18 & 22.8 \\
\hline
\end{tabular}

was a significant IP evaluation $x$ Short Answer Review interaction for the PCL-5, F $(1,1006)=19.77, p$ $<.001$. Post-hoc tests suggested that the responses of those who were valid based on IP evaluation and Short Answer Review had the lowest PCL-5 scores compared to the other groups ( $p$ 's $<.001$; Supplemental Table 1; Figure 2a). Furthermore, the mean score of the invalid responses was close to the center of the possible range of scores on the PCL-5 $(M=43.64$, Center $=40)$. This mean score is notable as it is likely to be obtained when choosing answers on the measure at random. A similar set of findings emerged for the PHQ-8. There was a significant IP evaluation $\times$ Short Answer Review interaction, $F(1,1006)=6.15, p=.013$ (Supplemental Table 1; Figure 2b). The mean PHQ-8 score of the invalid responses was also at the center of the range of scores $(M=13.21$, Center $=$ 13.50). Finally, the number of traumatic event types that were endorsed were compared across the evaluation methods. There was a significant IP evaluation $\times$ Short Answer Review interaction, $F(1,1006)=9.08, p=.003$. Those who were determined to be valid by both methods endorsed fewer traumatic event types than the other groups ( $p$ 's $<.001$; Supplemental Table 1; Figure $2 c$ ). Of note, 221 invalid responses endorsed every type of traumatic event on the LEC-5.

Responses were then compared between the Attention Check and Cyborg Methods. Of the 1011 responses who endorsed an event on the LEC, $n=890$ (88.03\%) completed all 6 attention check items correctly. A substantial portion of this subset $(n=655,64.8 \%)$ completed all 6 attention check items but did not pass the validity checks of the Cyborg Method. Alternatively, only 18 responses (1.8\%) were considered valid by the Cyborg Method, but not the Attention Check method. A $2 \times 2$ ANOVA (Cyborg $\times$ Attention Check) comparing PCL responses showed a significant interaction, $F(1,1006)=5.72 p=.017$. Post-hoc comparisons suggested that within the Attention Check group, those with valid Cyborg Method responses had significantly lower scores (diff $=17.28, p<$ 
Figure 2. Comparisons of Scores on the PCL-5 and PHQ-9 across the different categories of validation. Panel A: PCL-5 scores. Panel B: PHQ-9 Scores, Panel C: Number of Trauma Types Endorsed on the Life Events Checklist. Cyborg $N=232$. IP Evaluation $N=167$. Short Answer $N=256$. Invalid $N=324 . * * *=p<.001$. ** $=p$ $<.01$.

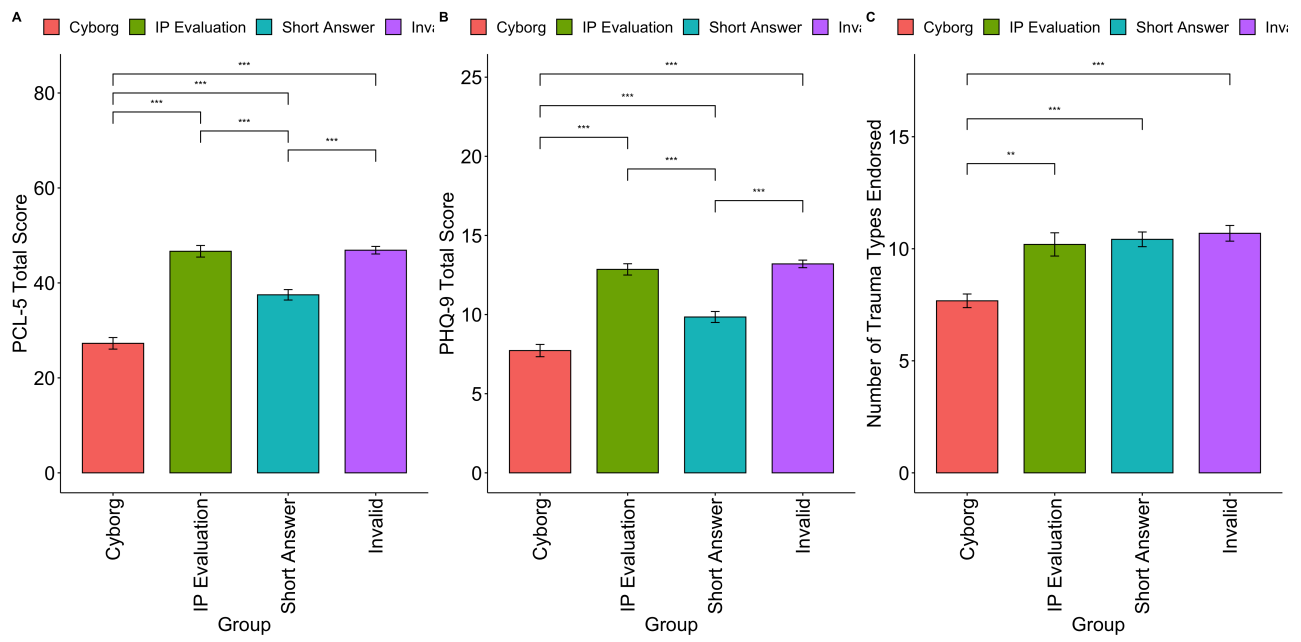

.001 , Figure 3). For PHQ-8 scores, there was a main effect for the Cyborg Method, $F(1,1006)=4.06$, $p=.044$. Post hoc tests suggested that those who were valid according to the Cyborg Method were significantly lower than those who were not (diff $=3.57, p<.001$ ). There was no main effect for the Attention Check group and no significant interaction. There was a significant main effect for the Cyborg Method for the number of trauma types, $F(1,1006)=45.56, p<.001$ (Figure 3). Again, this group endorsed fewer event types than those in the other groups. Taken together, these results suggest that the Cyborg Method was more likely to detect valid responses than the Attention Check method.

Figure 3. Comparisons of Scores on the PCL-5 and PHQ-9 across the Cyborg and Attention Check Methods. Panel A: PCL-5 scores. Cyborg Attention $\mathrm{N}=235$. Cyborg Only $\mathrm{N}=18$. Attention Only $\mathrm{N}=655$. Invalid $\mathrm{N}=235$. Panel B: PHQ-9 Scores, Panel C: Number of Trauma Types Endorsed on the Life Events Checklist. *** $=p<$ $.001 .{ }^{*}=p<.05$.

A Cyborg \& Attention $\square$ Cyborg Only $\square$ Attentic Legend_Att $\square$ Cyborg \& Attention $\square$ Cyborg Only $\square$ Legend_Att $\square$ Cyborg \& Attention $\square$ Cyborg Only $\square$ Attention
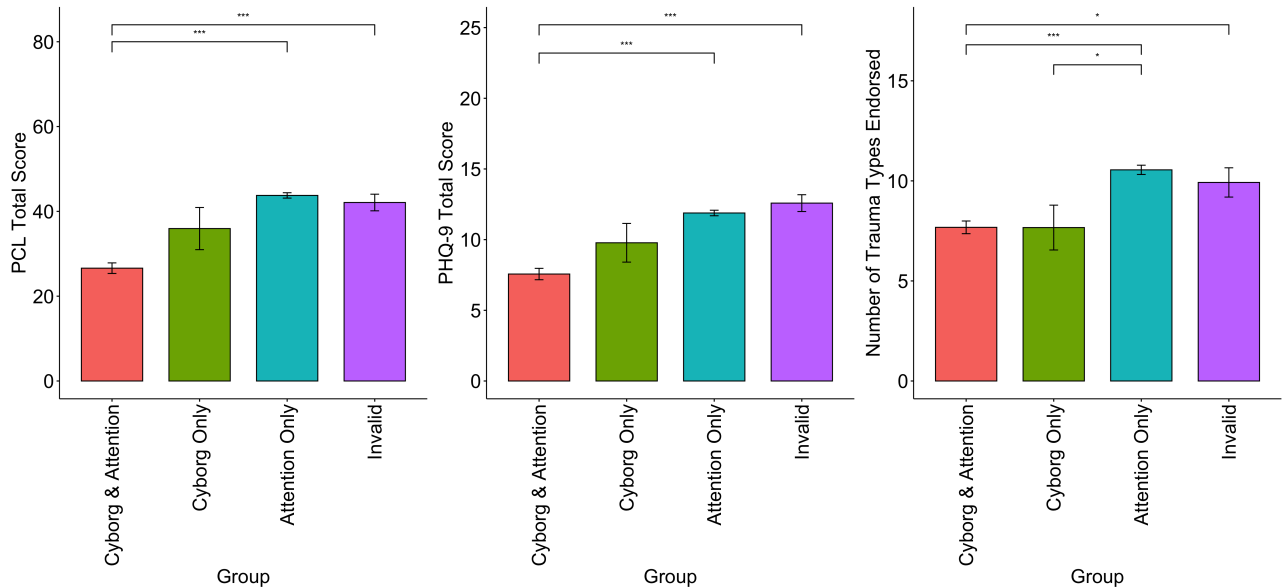


\section{Economic Impact}

The economic impact of the Cyborg Method was then evaluated. For Sample 1, 264 participants were determined to be valid by the Cyborg Method. A small subset $(32,12.1 \%)$ provided a valid short answer response that did not describe a traumatic event that would meet Criterion $A$ for PTSD. Thus, a valid sample of 232 participants was obtained for $\$ 4,900$, resulting in a cost per participant of $\$ 21.12$. This is an excess cost of (\$21.12 - \$4.80) $\$ 16.82$ per participant. Sample 2 was then recruited using a budget of $\$ 1,331$ and proactively applying the Cyborg Method (Table 2; Figure 1). Invalid responses were rejected when they were identified and not provided compensation. For this sample, 301 were considered valid by the Cyborg Method. Of these responses, 30 reported a stressful event that did not meet Criterion A for PTSD. The final sample of 271 participants had a cost of $\$ 4.91$ per participant, resulting in an excess cost of $\$ 0.11$ per participant. Thus, the cyborg method substantially reduce cost per participant.

\section{Sample 1 and Sample 2 Comparison}

The valid responses from Sample 1 and Sample 2 were then compared to determine if they differed. Given that these were recruited from the same population of MTurk participants with the same methods, it would be expected that the samples would be similar. This comparison was done by determining if there was an overlap in the $95 \%$ confidence intervals for the distributions of the PCL-5, PHQ-8, and LEC. If there was no overlap, a follow-up t-test was conducted to determine if the difference met the threshold for statistical significance at $p<.05$. For the PCL-5, the $95 \% \mathrm{Cl}$ for each sample did not overlap (Sample1: $M=27.05,95 \% \mathrm{Cl}: 24.53$ to 29.58; Sample 2: $\mathrm{M}=21.12$, $95 \% \mathrm{Cl}: 18.74$ to 23.49 ). A follow-up t-test confirmed that there was a significant difference between these groups, $\mathrm{t}(482)=3.37, \mathrm{p}=.001$. However, the mean difference between the samples was 5.94, which is relatively small. For the PHQ-8, the $95 \% \mathrm{Cl}$ for each sample overlapped (Sample 1: $\mathrm{M}=7.62$, $95 \% \mathrm{Cl}: 6.82$ to 8.42 ; Sample 2: $\mathrm{M}=6.56,95 \% \mathrm{Cl}: 5.76$ to 7.35 ), suggesting there was no difference between these samples (mean difference $=1.05$ ). Finally, there was overlap in the $95 \% \mathrm{Cl}$ for the number of traumatic event types endorsed in the two samples (mean difference $=0.82$; Sample1: $M=6.68,95 \% \mathrm{Cl}: 6.06$ to 7.31 ; Sample 2: $M=7.51,95 \% \mathrm{Cl}: 6.90$ to 8.11 ). The distributions for each of these measures were largely similar across samples (Supplemental Figure 1). Taken together, these results provide evidence that the Cyborg method replicated the collection of a valid sample of data. It also suggests this approach is cost effective when used proactively.

\section{Discussion}

The current study demonstrated the utility of a multimethod strategy to improve the quality of crowdsourced data that we are referring to as the Cyborg Method for its use of automated and human review of data. Prior work has shown that, when effective quality control measures are used, data collected via crowdsourcing is comparable to that of clinical samples collected via traditional means (Arch Carr, 2017; Engle et al., 2020; Morgan Desmarais, 2017; Shapiro et al., 2013; van Stolk-Cooke et al., 2018). The Cyborg Method is efficient in that it reduces investigator effort and financial costs, while increasing data quality. It also can be applied to previously collected data in which IP addresses and written responses were collected. Thus, it is a viable strategy for improving the quality of previously collected data as well as future data collection. The results of the present study highlight the need for multimethod evaluation of responses from crowdsourced data. Despite the high agreement between the IP evaluation and Short Answer Review, there were large subsets of responses that passed one evaluation but not the other. Those that passed the IP evaluation but failed the Short Answer Response Review may have used IP addresses that were newly acquired. Thus, the IP evaluation services were not aware of their fraud potential. Alterna- 
tively, several seemingly valid written responses came from invalid IP addresses. These written responses were vague descriptions of a trauma that appeared multiple times in the dataset. Detection of such responses by a researcher is challenging, but the IP evaluation service flagged them as problematic. Thus, it is highly recommended that the multimethod used in the present study be applied in future research using crowdsourced data. The proposed Cyborg Method has several advantages. First, it is reduces researcher burden such that the do not need to scour large crowdsourced datasets for invalid responses in that only a subset of responses with valid IP addresses needs to be considered for the Short Answer Review. The data from the Sample 1 showed that a substantial portion of participants with invalid IP addresses also provided invalid written responses. Second, the approach is flexible in its implementation. This method can be applied as needed during data collection or after study completion if all data needed to implement the method were collected. Finally, it is replicable as the data collected in Sample 2 was largely similar to that collected in Sample 1. There were no differences in depression scores, or the number of trauma types endorsed by both samples. The difference was relatively small between the two samples and the two distributions appeared relatively similar. Thus, there is reason to believe that continued use of this method would result in similar samples, especially among trauma-exposed individuals. The results of the present study also demonstrate the economic benefit of the Cyborg Method. The cost to recruit participants in Sample 1, where quality control measures were implemented after the data collection was completed, was four times that of Sample 2. This increased cost highlights the challenges of conducting crowdsourced research without proper quality control measures. For example, a study that was able to recruit $25 \%$ of its intended sample would likely draw erroneous conclusions from analyses due to the lack of power for the planned analyses. As crowdsourcing is likely to be used by early career investigators and students who may have limited financial resources to collect data, this budget efficiency is critical (Agley et al., 2021). The present study also compared the results of the "attention" check method of validating responses to the Cyborg Method. The majority of cases classified as valid by the Cyborg method were also classified as valid via the attention check method. However, a substantial number of responses that were invalid according to the Cyborg Method also answered all attention questions correctly, as was found in prior work (Dennis et al., 2019). This finding highlights the potential limitation of the attention check method for data validation. Respondents who submit invalid responses are likely aware of this approach and have strategies to navigate them (Chandler et al., 2020). Thus, relying on this strategy alone may still result in the collection of invalid data. Based on these results and the concern that the inclusion of such items may alienate certain users, researchers are recommended to adopt alternative strategies than attention check items (Vannette, 2017). The study had several limitations. First, the validity of each participant could not be verified independently. That is, we were unable to independently confirm that each response labeled valid was in fact valid and viceversa. As such, this method may still result in errors during data collection. It is recommended that an appeal process be included in such a data collection method to allow for incorrectly identified responses to be appealed to the study team and the outcomes rectified. Second, participants in the current study were paid 4 forcompletingthestudy, whichwasabovethe 2 market rate. This was done to incentivize participation, but it may have attracted more invalid responses. Finally, we were focused on collecting data from a trauma-exposed sample and thus the short answer questions were specific to trauma exposure. Future work should determine the extent that other short answer questions can be used as effectively as those about a traumatic event. In conclusion, the Cyborg Method provides an efficient method for obtaining high quality crowdsourced data. Methods that improve the quality of such data are needed to maintain the viability of this approach. Future work is needed to evaluate this method's utility and viability over time with a range of samples. This work should include novel methods to evaluate the qualitative Short Answer Review expedite that process. 'Bad actors' are likely to devise additional strategies to navigate existing quality control measure and further protections will be needed. If researchers and manuscript reviewers remain diligent, crowdsourcing will remain a viable method to collect data efficiently. 


\section{References}

[Qua]

[2] Agley, J., Xiao, Y., Nolan, R., and Golzarri-Arroyo, L. (2021). Quality control questions on amazon's mechanical turk (mturk): A randomized trial of impact on the usaudit, phq-9, and gad-7. Behavior Research Methods. [Online; accessed 2021-08-10].

[3] Aguinis, H., Villamor, I., and Ramani, R. S. (2021). Mturk research: Review and recommendations. Journal of Management, 47(4):823-837. publisher: SAGE Publications Inc.

[4] Arch, J. J. and Carr, A. L. (2017). Using mechanical turk for research on cancer survivors. Psycho-Oncology, 26(10):1593-1603. ${ }_{e}$ print : https : //onlinelibrary.wiley.com/doi/pdf/10.1002/pon.4173.

[5] Barnhoorn, J. S., Haasnoot, E., Bocanegra, B. R., and van Steenbergen, H. (2015). Qrtengine: An easy solution for running online reaction time experiments using qualtrics. Behavior Research Methods, 47(4):918-929.

[6] Boas, T. C., Christenson, D. P., and Glick, D. M. (2020). Recruiting large online samples in the united states and india: Facebook, mechanical turk, and qualtrics. Political Science Research and Methods, 8(2):232-250. publisher: Cambridge University Press.

[7] Bovin, M. J., Marx, B. P., Weathers, F. W., Gallagher, M. W., Rodriguez, P., Schnurr, P. P., and Keane, T. M. (2015), Psychometric properties of the ptsd checklist for diagnostic and statistical manual of mental disorders-fifth edition (pcl-5) in veterans. Psychological Assessment. PMID: 26653052.

[8] Buhrmester, M. D., Talaifar, S., and Gosling, S. D. (2018). An evaluation of amazon's mechanical turk, its rapid rise, and its effective use. Perspectives on Psychological Science, 13(2):149-154. publisher: SAGE Publications Inc.

[9] Chandler, J., Sisso, I., and Shapiro, D. (2020). Participant carelessness and fraud: Consequences for clinical research and potential solutions. Journal of Abnormal Psychology, 129(1):49-55. publisher-place: US publisher: American Psychological Association.

[10] Dennis, S. A., Goodson, B. M., and Pearson, C. A. (2019). Online worker fraud and evolving threats to the integrity of mturk data: A discussion of virtual private servers and the limitations of ip-based screening procedures. Behavioral Research in Accounting, 32(1):119-134.

[11] Dolan, M., Contractor, A. A., Ryals, A. J., and Weiss, N. H. (2020). Trauma, posttraumatic stress disorder severity, and positive memories. Memory (Hove, England), 28(8):998-1013. PMID: 32840463 PMCID: PMC7510933.

[12] Engle, K., Talbot, M., and Samuelson, K. W. (2020). Is amazon's mechanical turk (mturk) a comparable recruitment source for trauma studies? Psychological Trauma: Theory, Research, Practice, and Policy, 12(4):381-388. publisher-place: US publisher: Educational Publishing Foundation.

[13] Kroenke, K., Strine, T. W., Spitzer, R. L., Williams, J. B., Berry, J. T., and Mokdad, A. H. (2009). The phq-8 as a measure of current depression in the general population. Journal of Affective Disorders, 114(1-3):163-173.

[14] Maclnnis, C. C., Boss, H. C. D., and Bourdage, J. S. (2020). More evidence of participant misrepresentation on mturk and investigating who misrepresents. Personality and Individual Differences, 152:109603.

[15] Mellis, A. M. and Bickel, W. K. (2020). Mechanical turk data collection in addiction research: utility, concerns and best practices. Addiction, 115(10):1960-1968. ${ }_{e}$ print : https : //onlinelibrary.wiley.com/doi/pdf/10.1111/add.15032.

[16] Morgan, J. K. and Desmarais, S. L. (2017). Associations between time since event and posttraumatic growth among military veterans. Military Psychology, 29(5):456-463. publisher: Routledge ${ }_{e}$ print : https : //doi.org/10.1037/mil0000170.

[17] Natesan Batley, P., Contractor, A. A., Weiss, N. H., Compton, S. E., and Price, M. (2021). Psychometric evaluation of the posttrauma risky behaviors questionnaire: Item response theory analyses. Assessment, page 10731911211036760. publisher: SAGE Publications Inc.

[18] Shapiro, D. N., Chandler, J., and Mueller, P. A. (2013). Using mechanical turk to study clinical populations. Clinical Psychological Science, 1(2):213-220. publisher: SAGE Publications Inc.

[19] Strickland, J. C. and Stoops, W. W. (2019). The use of crowdsourcing in addiction science research: Amazon mechanical turk. Experimental and Clinical Psychopharmacology, 27(1):1-18. PMID: 30489114. 
[20] van Stolk-Cooke, K., Brown, A., Maheux, A., Parent, J., Forehand, R., and Price, M. (2018). Crowdsourcing trauma: Psychopathology in a trauma-exposed sample recruited via mechanical turk. Journal of Traumatic Stress, 31(4):549-557. ${ }_{e}$ print : https : //onlinelibrary.wiley.com/doi/pdf/10.1002/jts.22303.

[21] Vannette, D. (2017). Using attention checks in your surveys may harm data quality. [Online; accessed 2022 01-03].

[22] Weathers, F. W., Blake, D. D., Schnurr, P. P., Kaloupek, D. G., Marx, B. P., and Keane, T. M. (2013). The life events checklist for dsm-5 (lec-5).

[23] Weber, S. (2021). A step-by-step procedure to implement discrete choice experiments in qualtrics. Social Science Computer Review, 39(5):903-921. publisher: SAGE Publications Inc.

[24] Weiss, N. H., Schick, M. R., Contractor, A. A., Goncharenko, S., Raudales, A. M., and Forkus, S. R. (2021). Posttraumatic stress disorder symptom severity modulates avoidance of positive emotions among trauma-exposed military veterans in the community. Psychological Trauma: Theory, Research, Practice, and Policy, pages No Pagination Specified-No Pagination Specified. publisher-place: US publisher: Educational Publishing Foundation. 